\title{
Better resource utilisation and quality of care for ovarian cancer patients using internet-based pathology review
}

\author{
S Kommoss ${ }^{*}, 1$, F Kommoss ${ }^{2}$, J Diebold ${ }^{3}$, S Lax ${ }^{4}$, D Schmidt ${ }^{2}$, A Staebler ${ }^{5}$, A du Bois ${ }^{6}$ and J Pfisterer ${ }^{7}$ \\ ${ }^{1}$ Department of Women's Health, Tübingen University Hospital, Calwer Street 7, 72076 Tübingen, Germany; ${ }^{2}$ Institute of \\ Pathology, Referral Centre for Gynecopathology, A2/2, 68159 Mannheim, Germany; ${ }^{3}$ Luzerner Kantonsspital, Institute of \\ Pathology, 6000 Lucerne, Switzerland; ${ }^{4}$ LKH Graz West, Institute of Pathology, Göstinger Street 22, 8020 Graz, Austria; ${ }^{5}$ Tübingen \\ University Hospital, Institute of Pathology, Liebermeister Street 8, 72076 Tübingen, Germany; ${ }^{6}$ Department of Gynecology and \\ Gynecologic Oncology, Kliniken Essen Mitte, Henrici Street 92, 45136 Essen, Germany and ${ }^{7}$ Gynecologic Oncology Center Kiel, \\ Herzog-Friedrich-Street 21, 24103 Kiel, Germany
}

Background: The current literature indicates that a considerable number of patients in ovarian carcinoma clinical trials have histopathological diagnoses in conflict with inclusion criteria. It has been suggested that specialised pathology review prior to randomisation should become the standard procedure in study protocols. We hypothesised that our new, internet-based highthroughput infrastructure would be capable of providing specialised pathology review within 10 working days (w.d.).

Methods: Patients scheduled for the AGO OVAR17 ovarian carcinoma chemotherapy trial were registered for expert pathologic case review using a new internet-based central pathology review platform prior to randomisation. All original slides were requested from local pathologists. Slides were scanned and uploaded to a secured internet server. A network of experienced gynaecological pathologists was connected to the server through a custom-designed software platform. If deemed necessary by the expert pathologists, immunohistochemistry was available through a collaborating pathology lab.

Results: A total of 880 patients with an original diagnosis of ovarian epithelial carcinoma were registered for expert pathology review from October 2011 to July 2013. For case review, five gynaecopathologists from Austria, Switzerland and Germany were available online. Median number of w.d. required to complete the whole process from patient registration to transmission of final review diagnoses was 4 (range 2-31) (w.d.), and in 848 out of 880 (97.5\%) cases, it amounted to $\leqslant 10$ w.d. In 2.5\% ( $n=22$ ) of cases, a major diagnostic discrepancy of potential clinical relevance was found leading to exclusion from the chemotherapy trial.

Conclusions: Our results show that the use of a new internet-based infrastructure makes timely specialised case review, prior to patient randomisation feasible within $\leqslant 10$ w.d. Our new approach helped to protect against overtreatment with chemotherapy of patients with ovarian borderline tumours and inadequate treatment of patients with ovarian metastases, as a result of their inappropriate entry into a clinical trial designed for patients with primary ovarian carcinoma.

We recently reported that a considerable number of patients in a clinical trial of ovarian carcinoma had diagnoses other than primary epithelial carcinoma in conflict with inclusion criteria. Such pathological misdiagnoses in clinical studies might not only skew interpretation of study results, if not identified through central pathology review, but could also cause unnecessary treatment-related morbidity and might put a considerable strain on health-system budgets and society in general. It was concluded, that specialised central pathology review at the time of enrolment should be an integral part of future clinical studies of ovarian

*Correspondence: Dr S Kommoss; E-mail: stefan.kommoss@med.uni-tuebingen.de

Received 9 August 2016; revised 14 October 2016; accepted 17 November 2016; published online 22 December 2016

(c) 2017 Cancer Research UK. All rights reserved 0007-0920/17 
carcinomas (Kommoss et al, 2013). To meet study inclusion schedules, once a diagnosis of ovarian carcinoma is made, the process of pathological case review would have to be completed in a timely manner. However, the workflow for successful pathology review in the setting of large multicentre trials enrolling several hundred patients is very complex and has up to the present been very time-consuming. In the process of histopathological case review, a multitude of organisational steps are necessary including patient registration, requesting of original pathology reports, slides and blocks, case retrieval and shipment of such material, registration and distribution of received material among experts, histopathological review possibly necessitating additional workup, as well as documentation and distribution of expert diagnoses. Hitherto, the complexity of this process has made it difficult if not impossible to complete case review within the limited time window before randomisation. It was the aim of this study to establish a new and more effective infrastructure that might considerably speed up central pathology review. We hypothesised that the review platform described in this paper might allow us to provide review diagnoses necessary for study enrolment purposes within 10 working days (w.d.). A secondary aim of this study was to investigate discrepancy rates and the implications of those discrepancies in a large multicentre clinical trial setting using our new internet-based review platform.

\section{MATERIALS AND METHODS}

A central study office staffed with two secretaries providing continuous day-time service during workdays was set up. Office hardware consisted of an Aperio Slide Scanner with hooked-up internet slide server, two computer workstations, telephone, fax, email and broadband internet facilities.

For case review, an international panel of five established gynaecologic pathologists was recruited (JD, FK, SL, DS and AS). For better future comparability of results, all experts agreed to review cases using the WHO 2014 diagnostic criteria, which were already accessible to the panel. Each of the pathologists was provided with a high-resolution monitor (Hewlett Packard, Palo Alto, CA, USA; LP2475w) for optimal visualisation of scanned slides. To cover additional workup of cases as deemed necessary by the experts, a pathological laboratory was contracted. A customdesigned internet platform and database were developed linking together the central office and all experts, as well as providing an interface for pathology review requests. Overnight transportation of pathology materials was secured by an express courier service (Figure 1).

Histopathological case review prior to randomisation using our new platform was made a mandatory integral part of the AGO OVAR17 trial according to OVAR17 translational subprotocol AGO VISION-OVAR. The OVAR17 trial evaluates optimal initial treatment duration of bevacizumab in combination with standard chemotherapy, NCT01462890. Inclusion criteria were: primary diagnosis of epithelial ovarian, fallopian tube or peritoneal carcinoma, FIGO stage IIB-IV, all histological types and grades.

Standard VISION-OVAR procedures were as follows: any patient considered for AGO OVAR17 study enrolment had to be registered for review of original pathological diagnosis after informed consent. To do so, clinical departments wishing to have a patient enrolled into the AGO OVAR17 trial had to contact our central study office by telephone, fax or internet. In addition to a patient registration form, the original pathology report, as well as the signed patient consent form had to be provided and were uploaded to our internet review platform. Upon completion of patient registration, an automated fax request was sent to the local pathologist responsible for the original diagnosis. The fax message

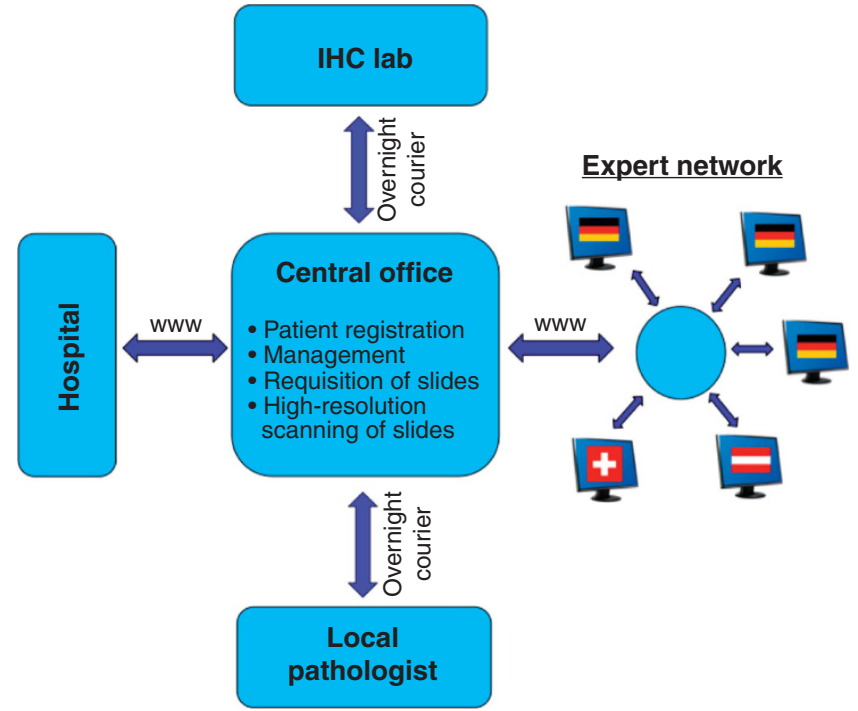

Figure 1. Internet-based infrastructure of the VISION-OVAR ovarian carcinoma second-opinion pathology network.

informed the pathologist about the planned OVAR17 study enrolment necessitating a fast central pathology review according to the VISION-OVAR subprotocol. The pathologist was asked to retrieve all histological slides including all immunostains and to have all materials ready for prepaid pickup, during the following w.d. EUR 35-reimbursement for case retrieval costs was offered. An additional telephone call and provision of appropriate shipping forms by our secretarial staff were intended to secure transportation of local pathology material to our central study office as soon as possible. Once case retrieval was confirmed, overnight courier service was initiated by our central study office staff members.

Once the shipment of a given case had arrived at the central office, secretarial staff members selected slides to be scanned for review. Staff members were instructed to thoroughly read the original pathology report. From each given listing of slides in those reports, they were to simply select those slides that were described as containing tumour tissue. Prior to study initiation, staff members had been made sufficiently familiar with pathological terminology referring to presence of tumour. Staff members were asked to aim for the selection of five suitable tumour-bearing slides per case. This number of slides had been determined based on previous experience in a large study of pathology review of ovarian carcinomas (Kommoss et al, 2013). In cases where $<5$ tumourbearing slides were listed in the pathology report, all tumourbearing slides were selected. If more than five such slides were listed, staff members were instructed to choose the five supposedly best suitable slides taking into consideration the given locations and descriptions in the pathology report. Selected slides were then scanned at $\times 20$ magnification and uploaded to the internet slide server. Upon completion of slide upload, an expert was electronically selected following a repetitive numerical order, who then received an automatically generated review invitation email. Within the following $24 \mathrm{~h}$, the invited expert could accept or decline case review without delay. If the expert declined or did not respond within $24 \mathrm{~h}$, the internet platform automatically selected and invited the next expert in line.

Upon accepting to review a case, the expert had access to the original path report, to the uploaded slide scans, as well as to information about all additional slide material available. As a first obligatory review step, the expert entered the 'original diagnosis' after reading the local pathologist's report. As for serous carcinoma types, this step involved translating original diagnoses still applying the WHO 2003 terminology (serous carcinoma, G1-G3) into 
WHO 2014 terminology. Any original serous carcinoma, G1 was considered low-grade serous ovarian carcinoma (LGSC), and any original serous carcinoma, G2-3 was logged as high-grade serous ovarian carcinoma (HGSC). Thereafter, slides were evaluated on the high-resolution monitor using the Aperio Image Viewer software (Leica Biosystems, Vista, CA, USA), which accessed the central office slide server via broadband internet connection. If having evaluated the selected slides, the expert was able to completely confirm the original diagnosis or to at least confirm the diagnosis of ovarian epithelial carcinoma, however, with a different histological type (minor discrepancy, not preventing OVAR17 enrolment), the case review was considered completed. After logging the review diagnosis, three letters were generated automatically and were to be electronically signed by the expert. The first letter was sent by fax or email to the clinical department involved indicating that AGO OVAR17 patient randomisation was now possible after completion of expert pathology review excluding any diagnosis other than primary epithelial ovarian carcinoma (major discrepancy). Simultaneously, the AGO study group overseeing the AGO OVAR17 trial was also notified in a second letter containing the anonymised review data. Third, a pathological consultation report containing the reviewer's name and contact information was printed out in our central office and sent to the local pathologist together with all submitted materials.

If having reviewed the first set of slides, the expert was unable to make a final diagnosis, several options were available through the platform. The pathologist could choose to have additional selected slides scanned and uploaded at either standard or $\times 40$ magnification. If deemed necessary, the expert could choose a certain slide and request to have $\mathrm{H} \& \mathrm{E}$ slides recut and/or immunohistochemical stains performed in the contracted pathology laboratory. As soon as an immunostaining request was logged, appropriate paraffin block acquisition was automatically initiated and executed by our central office following the same procedures as described above for the initial slide request. Paraffin blocks were directly delivered to the collaborating laboratory, which had already been informed about the pending immunostaining request by an automatically generated work order. Immunostains were performed within the w.d. following arrival. The requested stainings were performed following the standard operating procedures including a quality-control $\mathrm{H} \& \mathrm{E}$ section and appropriate controls. Express courier-service delivery of blocks and immunostains to our central office was initiated after sign-out by the laboratory's pathologist on duty. The received immunostains were scanned and uploaded, and the requesting expert was subsequently notified by email. Another option available to the reviewing expert was sharing the case in question with other panel experts for online consultation through our platform.

If after completion of one or even several of the above described measures, the reviewer's final diagnosis was to confirm the original diagnosis or to diagnose a minor discrepancy only, termination of the review process and notification of all partners involved ensued as already described. However, if the expert's final review diagnosis was other than epithelial ovarian carcinoma, he logged a major discrepancy. This set off action as predefined in the VISIONOVAR study protocol. A consultation letter suggesting a possible major discrepancy proposing case discussion between a local pathologist and a reviewing expert was generated, and electronically signed by the expert. For documentation purposes and to facilitate case discussion, all slides that had up to that moment not yet been scanned were uploaded to our slide server. All original material provided by the local pathologist was then returned to the local pathologist together with a printout of the consultation letter. Soon thereafter, the review pathologist called the local pathologist to discuss the case in question. If a final consensus diagnosis was reached, the arguments upon which it was based were documented in our database, and the clinical department as well as the AGO study group were notified as described above. The study protocol allowed for a maximum of three phone calls intended to make case discussion between the reviewer and local pathologist possible. If after case discussion, no consensus could be reached or if the local pathologist was consistently unavailable for case discussion, all other experts were requested to review the case over the internet platform, and the final diagnosis was to be reached by majority vote of the expert panel (Figure 2). This final panel diagnosis was supposed to be documented in a detailed consultation letter, which was sent to the local pathologist. Again, the clinical department as well as the AGO study group were notified.

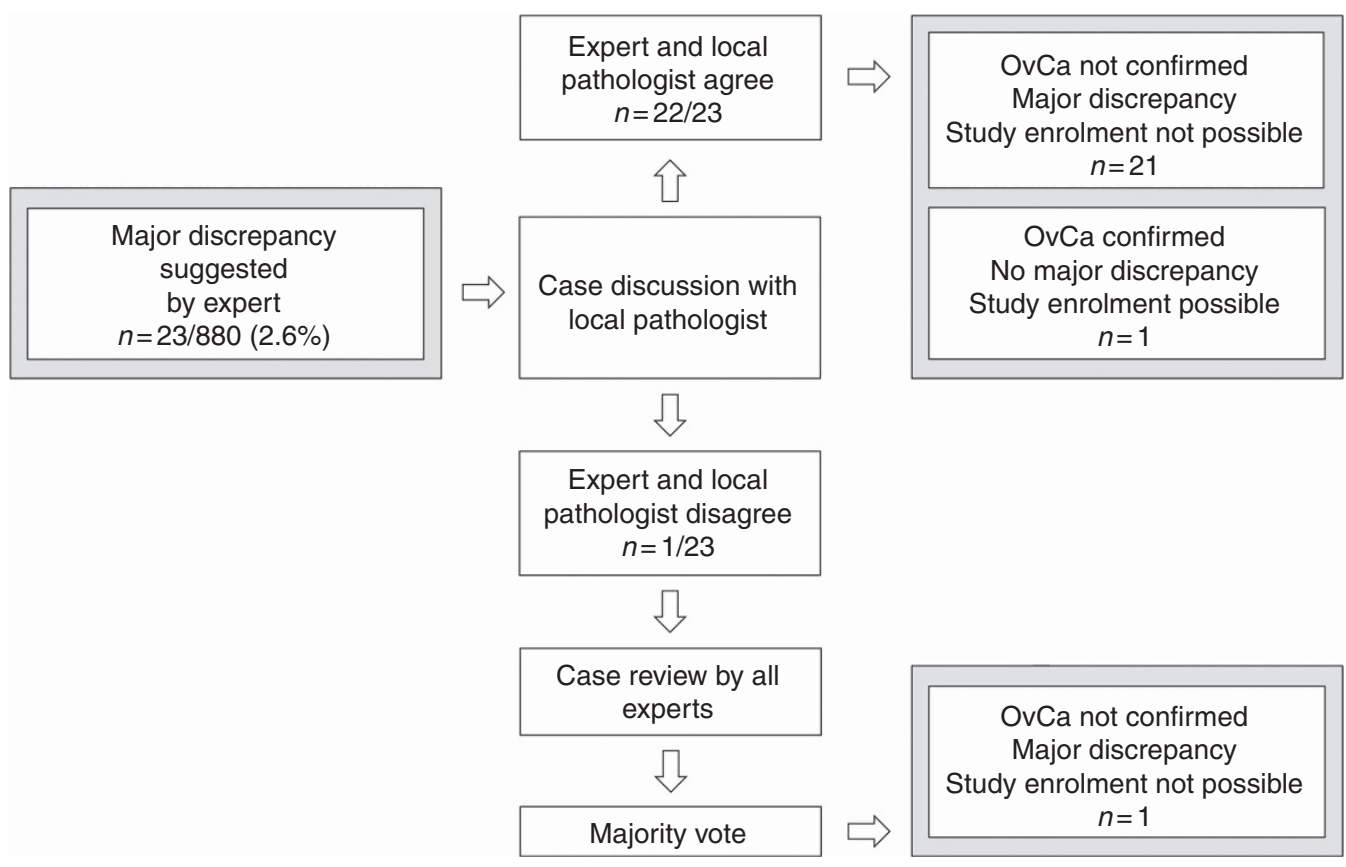

Figure 2. Workflow and results in cases where major discrepancies were suggested by expert pathologists. 
Statistical analyses were performed using JMP software (JMP, SAS Institute Inc., Cary, NC, USA, 1989-2007), our algorithm to calculate w.d. involved subtracting Saturdays, Sundays and German statutory holidays from total days.

\section{RESULTS}

During AGO OVAR17 recruitment (October 2011-July 2013), 110 clinical departments (AGO OVAR17 study centres) requested pathology review according to the VISION-OVAR study protocol for a total of 880 patients. During the study, a total of 44.198 histological slides (including 2.232 immunohistochemical stains of 93 different immunomarkers from 335 cases) were received from 132 different local pathology departments (average: 50 slides per case; range 1-185). During case review, 6.454 slides were scanned at $\times 20$ magnification (average 7.3 slides per case).

In 751 out of $880(85 \%)$ cases, mere review of on average 5 slides (range 1-30) selected by the central office staff was sufficient for completion of expert review. In the remaining 129 cases (15\%), additional workup as requested by the experts was necessary, increasing the number of slides reviewed to an average of 21 slides per case (range 4-101). Up to three rounds of additional standard magnification scans were requested in 112 out of 129 (87\%) cases, additional high magnification $(\times 40)$ scans were requested in 5 out of $129(4 \%)$ cases. Immunohistochemical stains were requested by the experts in 30 out of $129(23 \%)$ cases (including 124 immunohistochemical stains of 32 different immunomarkers). Additional scans as well as immunostains were requested in 15 out of $129(12 \%)$ cases.

Median number of w.d. required to complete the whole process from patient registration to transmission of final review diagnoses was 4 (range 2-31) w.d., and in 848 out of 880 (97.5\%) cases, it amounted to $\leqslant 10$ w.d. The whole process was found to consist of two major components, the first one being case retrieval and distribution to experts (median 4 w.d., range 2-23 w.d.), a detailed breakdown of which is given in Table 1 .

The second component comprised the actual expert case review (median 1 w.d., range 1-28 w.d.). If no additional workup was needed, case review was completed within a median of 1 w.d. (range 1-8 w.d.). As expected, total review time was longer in cases where additional workup was necessary (with additional scans: median 2 w.d., range 1-28 w.d.; with immunohistochemistry: median 8 w.d., range 5-21 w.d.).

The distribution of histological types of ovarian carcinomas as per original diagnoses and after VISION-OVAR case review is shown in Table 2.

The percentages of carcinoma histotypes were found to be almost identical, however, after comparison on a case per case basis, agreement rates varied between $30 \%$ in undifferentiated and $92 \%$ for HGSC. Overall, 732 out of 880 (83.2\%), original diagnoses were confirmed. Minor discrepancies were found in 126 out of 880 $(14.3 \%)$. Major discrepancies were suggested in 23 out of 880 (2.6\%) cases by the experts, of which 22 out of 880 (2.5\%) cases were finally confirmed after case discussion with local pathologists (Figure 2). The lesion most frequently mistaken for a primary epithelial ovarian carcinoma was carcinoma metastatic to the ovary ( $n=13$ out of 22 ), of which 11 out of 13 were assumed to originate in the gastrointestinal tract, and one each in the uterine cervix and in the endometrium. The second most frequent misdiagnosis was serous borderline tumour ( $n=7$ out of 22). Finally, one mixed germ cell tumour and one adenosarcoma were misdiagnosed as ovarian epithelial carcinoma (Table 3).

In all but one of the 22 major discrepancies, a final diagnostic consensus was reached after consultation with the local pathologist who after case discussion conceded the diagnosis as suggested by
Table 1. Detailed breakdown of steps necessary for case retrieval and number of working days during which each step was completed

\begin{tabular}{|l|c|c|c|}
\cline { 2 - 4 } & \multicolumn{3}{c|}{ Working days } \\
\cline { 2 - 4 } & Median & Min & Max \\
\hline Patient registration & 1 & 1 & 14 \\
\hline $\begin{array}{l}\text { Preparing shipment of slides at local pathology } \\
\text { departments }\end{array}$ & 2 & 1 & 16 \\
\hline Courier shipment & 2 & 1 & 8 \\
\hline Selection and scanning of slides at central office & 1 & 1 & 5 \\
\hline Expert assignment & 1 & 1 & 4 \\
\hline
\end{tabular}

Table 2. Distribution of histological types of ovarian

carcinomas as per original diagnoses and after VISION-OVAR case review

\begin{tabular}{|c|c|c|c|c|c|c|}
\hline \multirow[b]{2}{*}{$\begin{array}{l}\text { Histopathological type } \\
(n=880)\end{array}$} & \multicolumn{4}{|c|}{$\begin{array}{l}\text { Original } \text { Review } \\
\text { diagnosis diagnosis }\end{array}$} & \multicolumn{2}{|c|}{$\begin{array}{l}\text { Agreement } \\
\text { on review }\end{array}$} \\
\hline & $\mathbf{n}=$ & $\%$ & $\mathbf{n}=$ & $\%$ & $\mathbf{n}=$ & $\%$ \\
\hline $\mathrm{HGSC}^{\mathrm{a}}$ & $701^{b}$ & 79.6 & 701 & 79.6 & $648 / 701$ & $92 \%$ \\
\hline LGSC & $47^{c}$ & 5.3 & 48 & 5.5 & $38 / 47$ & $80 \%$ \\
\hline EC & 33 & 3.8 & 16 & 1.8 & $12 / 33$ & $36 \%$ \\
\hline UC & 23 & 2.6 & 9 & 1.0 & $7 / 23$ & $30 \%$ \\
\hline Mixed & 22 & 2.5 & $52^{d}$ & 5.9 & $11 / 22$ & $50 \%$ \\
\hline $\mathrm{CCC}$ & 12 & 1.4 & 13 & 1.5 & $7 / 12$ & $58 \%$ \\
\hline MC & 11 & 1.3 & 4 & 0.5 & $4 / 11$ & $36 \%$ \\
\hline MMMT & 11 & 1.3 & 12 & 1.4 & $9 / 11$ & $82 \%$ \\
\hline TCC & 3 & 0.3 & 3 & 0.3 & $1 / 3$ & $33 \%$ \\
\hline No type & 17 & 1.9 & - & - & - & - \\
\hline Other than ovarian carcinoma & - & - & 22 & 2.5 & - & - \\
\hline \multicolumn{7}{|c|}{ 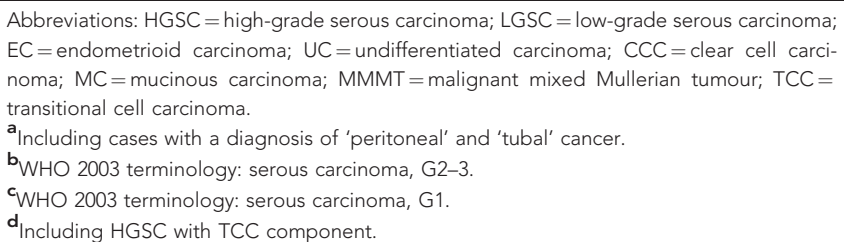 } \\
\hline
\end{tabular}

the expert. In only one case did the local pathologist insist on their original diagnosis of primary epithelial ovarian carcinoma even after case discussion, and the final review diagnosis had to be made by majority vote of the expert panel, which unanimously endorsed the presence of a major discrepancy. In one case, the review pathologist had suggested a major discrepancy, however, the original diagnosis was finally confirmed after consultation of the local pathologist who then provided important additional slides and block material allowing for an unequivocal diagnosis of primary ovarian carcinoma (Figure 2).

The complexity of the review process markedly increased if minor or major discrepancies were noted, as mirrored by a higher percentage of cases with additional scans (no discrepancy: 10\%, minor discrepancies: $18 \%$ and major discrepancies: $73 \%$ ) and/or immunohistochemistry (no discrepancy: 1\%, minor discrepancies: $7 \%$ and major discrepancies: $50 \%$ ). Of note, local pathologists had used immunohistochemical stains in 15 out of $22(68 \%)$ cases with major discrepancies. Furthermore, often lengthy consultation with the local pathologist was an integral part in all cases with major discrepancies. As a result, case review time was significantly increased in cases where major discrepancies were found (median 
Table 3. Major discrepancies as found in 22 (2.5\%) of 880 cases

\begin{tabular}{|c|c|c|}
\hline ID & Original diagnosis & Review diagnosis \\
\hline 176 & 'Peritoneal cancer' & Adenocarcinoma of gastrointestinal tract, metastatic to the peritoneum \\
\hline 387 & 'Peritoneal cancer' & SBOT of the peritoneum \\
\hline 516 & 'Peritoneal cancer' & Pancreatobiliary-type adenocarcinoma, metastatic to the peritoneum \\
\hline 793 & 'Peritoneal cancer' & Adenocarcinoma of gastrointestinal tract, metastatic to the peritoneum \\
\hline 852 & 'Peritoneal cancer' & Endometrial adenocarcinoma, serous, metastatic to the peritoneum \\
\hline 61 & HGSC & SBOT, micropapillary pattern \\
\hline 130 & HGSC & SBOT, non-invasive implants \\
\hline 208 & HGSC & Adenocarcinoma of gastrointestinal tract, metastatic to the ovary \\
\hline 862 & HGSC & Cancer of unknown primary (most likely gastrointestinal tract), metastatic to the ovary \\
\hline 909 & HGSC & Adenocarcinoma of gastrointestinal tract, metastatic to the liver \\
\hline 252 & MC & Endocervical adenocarcinoma, metastatic to the ovary \\
\hline 258 & MC & Adenocarcinoma of gastrointestinal tract, metastatic to the ovary \\
\hline 470 & MC & Adenocarcinoma of gastrointestinal tract, metastatic to the ovary \\
\hline 775 & MC & Adenocarcinoma of gastrointestinal tract, metastatic to the ovary \\
\hline 552 & LGSC & SBOT, focal microinvasion, lymph node involvement \\
\hline 669 & LGSC & SBOT, non-invasive implants in parametria and lymph nodes \\
\hline 172 & $\mathrm{CCC}$ & Malignant mixed germ cell tumour of the ovary \\
\hline 756 & $\mathrm{CCC}$ & Adenocarcinoma of gastrointestinal tract, metastatic to the ovary \\
\hline 103 & $\mathrm{EC}$ & Adenocarcinoma of gastrointestinal tract, metastatic to the ovary \\
\hline 88 & MMMT & Adenosarcoma with sarcomatous overgrowth \\
\hline 87 & Mixed & SBOT, peritoneal implants \\
\hline 238 & No type given & SBOT, micropapillary pattern, invasive and non-invasive implants \\
\hline
\end{tabular}

duration of case review with no or minor discrepancy: 4 w.d.; with major discrepancy: 11.5 w.d.).

It was not possible to provide review diagnoses within 10 w.d. in 31 out of $880(2.5 \%)$ cases. The three contributing factors were: finding of a major discrepancy (12 out of 31), delays caused by lengthy registration at the clinical departments or case retrieval at the local pathology departments (11 out of 31 ), and complex case review (8 out of 31 ).

\section{DISCUSSION}

Most therapeutic clinical trials require patient enrolment and initiation of treatment within a set time frame after a diagnosis has been made. In the setting of advanced-stage primary ovarian carcinoma, it is recommended to start post-operative adjuvant chemotherapy within 4-6 weeks after radical upfront debulking surgery (Lydiksen et al, 2014). Moreover, when neoadjuvant chemotherapy is considered, and surgery is limited to less radical diagnostic procedures only, it is usually possible to initiate chemotherapy even sooner. It has been common practice to enrol patients with ovarian cancer to clinical trials based on the original histopathological diagnoses without routine case review. In the future, pretreatment confirmation of pathological diagnoses may become more and more desirable considering our recent findings of clinically relevant diagnostic discrepancies in a clinical trial patient cohort and in the light of the upcoming era of modern histotype-specific ovarian carcinoma treatment (Kobel et al, 2008; McCluggage, 2011; Prat, 2012; Kommoss et al, 2013). The results of this study may illustrate a mechanism to implement timely secondopinion pathology as an integral step in future study protocols.

Using our newly created internet-based review platform, we were able to provide clinical departments with pathology review data within 10 w.d. for 848 out of 880 (97.5\%) patients considered for AGO OVAR17 enrolment. The privilege of working together in a highly motivated team helped accomplish our study goals; availability and review time of panel experts were not a concern in our study setting. With that component in place, the paramount factor for successful platform implementation was the creation of a custom-design internet-based software platform linking a permanently staffed central office to clinical departments, local pathologists and an international panel of gynaecopathological experts. In addition, centralised handling of all materials, digital slide access, software algorithms keeping track of complex organisational processes and the use of express courier services were key factors. Feedback from all collaborating partners involved was mostly encouraging. Strain was taken off clinical departments not having to assist with case retrieval, and local pathologists considered a streamlined case retrieval process helpful, many expressing gratitude for expert feedback and optional personal consultation. The panel experts' workload was considerably reduced by not having to deal with shipment and handling of materials, and by being relieved through the digital platform of having to provide full written reports themselves. The opportunity for case conferences and intercollegial exchange was also greatly appreciated by all experts.

This study confirms a benefit of pretreatment second-opinion pathology for ovarian carcinoma patients willing to participate in a chemotherapy trial. Performing pathology review helped avoid less than optimal treatments for patients with malignant tumours other than primary epithelial ovarian carcinoma, such as ovarian metastases and germ cell tumours. In addition, unwanted treatment-associated morbidity in patients with ovarian neoplasms not requiring post-operative chemotherapy such as ovarian borderline tumours could be avoided. Of note, the rate of major discrepancies $(2.5 \%)$ was markedly lower as compared with our previously reported discrepancy rate $(6.8 \%)$ in another study 
collective where pathology review had been performed without affecting actual patient treatment (Kommoss et al, 2013). It is interesting to speculate whether increasing general awareness among gynaecologic oncologists and pathologists about the importance of reliably diagnosing malignant ovarian tumours may have contributed to this highly desirable result.

The development and maintenance of our platform as well as costs associated with each individual case were financed within the AGO OVAR17 study budget. Having created this internet-based review platform could facilitate the implementation of desirable rapid pathology review into clinical trial study protocols. However, the future source of funding is as yet unclear, and the exact amount of money required for implementing similar review platforms into other study protocols cannot be foreseen with certainty as it is highly dependant on a multitude of individual factors (e.g., case volume, preexisting infrastructure including availability of slide scanners, volume and complexity of additional workup). To give a general idea of the financial prerequisites for implementing a review platform similar to the one described in this paper, total funding available for this project amounted to roughly EUR 320 per case. Almost two thirds of the total budget was spent on platform development and maintenance, both required to get the platform started, but unrelated to the actual number of case reviews performed. The remaining costs were directly associated with each individual case (such as case retrieval, reimbursement of local pathology departments, courier services, reimbursement of panel experts and immunohistochemistry).

There are certain limitations to our study. To accommodate the spectrum of ovarian carcinoma types widely used at the time of AGO OVAR17 patient recruitment, our study had to represent transition between WHO 2003 and WHO 2014 classifications of ovarian tumours. Although some lesions mentioned in our data set (e.g., transitional cell carcinoma) are no longer diagnosed according to WHO 2014, others (such as low- and high-grade serous carcinoma) have only recently been formally introduced after publication of the new classification system (Tavassoli, 2003; Kurman et al, 2014). This reflects the ongoing evolution of diagnostic criteria in ovarian carcinoma pathology. In order not to miss valuable scientific evidence when studying similar tumour collectives from the pre-WHO 2014 era, it may be necessary to revisit them, reviewing the histopathology slides and applying current diagnostic criteria (Kommoss et al, 2016). Furthermore, the review algorithm of our platform has its limitations, and there will most likely always be a small number of cases, which owing to their complexity cannot be successfully reviewed within up to 10 w.d. In addition, it is important to acknowledge that certain problematic factors associated with pathological case review, such as the willingness or ability of pathology departments to provide material in a timely manner cannot be influenced from within a platform such as the one described herein. Finally, a review process such as described herein is generally limited by available expert time. Although it might have been desirable to have two or more review pathologists look at each and every case, a second expert panelist was to be consulted only in a minority of difficult cases according to our study protocol.

Looking at the surprisingly low major discrepancy rate of only $2.5 \%$ in our current study, one might feel encouraged about the current high standard of histopathological diagnoses in the pathology community and might even question the justification of an elaborate review platform as described in our paper. However, it is important to realise that in addition to securing a diagnosis of epithelial carcinoma, confirmation of histotype will most likely become a key task of pretreatment pathology review in the upcoming era of histotype-specific ovarian carcinoma treatment (McAlpine et al, 2009; Anglesio et al, 2013), and minor discrepancies as described in this paper could be major discrepancies affecting patient treatment in the foreseeable future, in a histotype-specific clinical trial. A few such trials restricted to the typically chemoresistant low-grade serous, clear cell or mucinous histotype are already recruiting patients, and inclusion of a patient with HGSC in any of these trials would be a major discrepancy. Applying this thread of thought to our study collective, a significantly higher total of 148 out of 880 (16.8\%) patients would have had an altered diagnosis potentially affecting study enrolment and treatment decisions.

In conclusion, we have demonstrated proof of principle, that timely central expert pathology review prior to patient randomisation can be incorporated into clinical trials, with demonstrable benefits for patients whose diagnosis is changed based on review.

\section{CONFLICT OF INTEREST}

The authors declare no conflict of interest.

\section{REFERENCES}

Anglesio MS, Kommoss S, Tolcher MC, Clarke B, Galletta L, Porter H, Damaraju S, Fereday S, Winterhoff BJ, Kalloger SE, Senz J, Yang W, Steed H, Allo G, Ferguson S, Shaw P, Teoman A, Garcia JJ, Schoolmeester JK, Bakkum-Gamez J, Tinker AV, Bowtell DD, Huntsman DG, Gilks CB, McAlpine JN (2013) Molecular characterization of mucinous ovarian tumours supports a stratified treatment approach with HER2 targeting in 19\% of carcinomas. J Pathol 229(1): 111-120.

Kobel M, Kalloger SE, Boyd N, McKinney S, Mehl E, Palmer C, Leung S, Bowen NJ, Ionescu DN, Rajput A, Prentice LM, Miller D, Santos J, Swenerton K, Gilks CB, Huntsman D (2008) Ovarian carcinoma subtypes are different diseases: implications for biomarker studies. PLoS Med 5(12): e232.

Kommoss S, Gilks CB, du Bois A, Kommoss F (2016) Ovarian carcinoma diagnosis: the clinical impact of 15 years of change. Br J Cancer 115(8): 993-999.

Kommoss S, Pfisterer J, Reuss A, Diebold J, Hauptmann S, Schmidt C, du Bois A, Schmidt D, Kommoss F (2013) Specialized pathology review in patients with ovarian cancer: results from a prospective study. Int J Gynecol Cancer 23(8): 1376-1382.

Kurman RJ, Carcangiu ML, Herrington CS, Young RH (2014) WHO Classification of Tumours of Female Reproductive Organs. 4th edn. IARC Press: Lyon.

Lydiksen L, Jensen-Fangel S, Blaakaer J (2014) Is it possible to define an optimal time for chemotherapy after surgery for ovarian cancer? Gynecol Oncol 133(3): 454-459.

McAlpine JN, Wiegand KC, Vang R, Ronnett BM, Adamiak A, Kobel M, Kalloger SE, Swenerton KD, Huntsman DG, Gilks CB, Miller DM (2009) HER2 overexpression and amplification is present in a subset of ovarian mucinous carcinomas and can be targeted with trastuzumab therapy. BMC Cancer 9: 433.

McCluggage WG (2011) Morphological subtypes of ovarian carcinoma: a review with emphasis on new developments and pathogenesis. Pathology 43(5): 420-432.

Prat J (2012) New insights into ovarian cancer pathology. Ann Oncol 23(Suppl 10): x111-x117.

Tavassoli FA, Devilee P (2003) Pathology and Genetics of Tumours of the Breast and Female Genital Organs. IARC Press: Lyon.

This work is published under the standard license to publish agreement. After 12 months the work will become freely available and the license terms will switch to a Creative Commons AttributionNonCommercial-Share Alike 4.0 Unported License. 